$s \quad=$ Laplace transform variable

$t=$ time

$t_{s}=$ settling time

$T_{1}=$ magnitude of analyzer sampling period

$T_{2}=$ magnitude of transport lag

$T_{3}=$ magnitude of analysis lag

$x=$ concentration of component $A$ in effluent

$x_{i}=$ concentration of component $A$ in feed

$\theta=$ temperature of reactant mass

$\tau_{a}, \tau_{b}=$ reactor time constants

\section{Subseripts}

$s s \quad=$ steady state values

\section{LITERATURE CITED}

1. Huber, C. I., Doctoral Dissertation, Carnegie Institute of Technology, Pittsburgh, Pa. (1967).

2. Kermode, R. I., and W. F. Stevens, Can. J. Chem. Eng,, 81 (1961).

3. Lupfer, D. E., and M. W. Oglesby, ISA TRANS., 1, No. 1, 72 (1962).

4. Min, H. S., and T. J. Williams, Chem. Eng. Progr. Symposium Ser. No. 36, 57, 100 (1961).

5. Reswick, J. B., Trans. Am. Soc. Mech. Engrs., 78, No. 1, 153 (1956).

6. Ross, C. W., ISA TRANS., 2,69 (1963).

7. Smith, O. J., ISA Journal, 6, No. 2, 28 (1959).

8. Williams, T. J., and H. S. Min, ibid., No. $9,89$. Manuscript received May 8, 1968; revision received March 7, 1969;
paper accepted March 12, 1969. Paper presented at AIChE Tampa meeting.

\title{
Optimal Control of a Continuous Flow Stirred Tank Chemical Reactor
}

\author{
MARTIN A. JAVINSKY and ROBERT H. KADLEC \\ University of Michigan, Ann Arbor, Michigan
}

The time optimal control problem for jocket cooled continuous flow stirred tank reactor (CSTR) with an exothermic, irreversible, second-order, homogeneous, liquid-phase reaction (the saponification of ethyl acetate) was solved with the maximum principle and phose plane analysis. Both experimental studies and analogue computer simulation studies were conducted.

The overall performance of the experimental system agreed very well with the performance of the corresponding system simulated on an analogue computer. However, there were enough differences in the observed and predicted operating states ond switching curves to warrant the conclusion that the experimental performance can be significantly improved if experimental results are used to modify the results predicted with computer analysis. These differences were attributed to uncertainties in the model and the values of the model parameters as well as nonrandom (and unforeseen) measurement errors.

In recent years, the chemical engineering journals have contained an increasing number of articles on optimization and optimal control. Nearly all of these papers have been theoretical in nature. When applications of the theory are presented, they usually involve only the study of a system simulated on a digital or analogue computer. The words "data" and "results" are only rarely preceded by the word "experimental;" instead, computer data and computer results for systems simulated on a computer are used to verify theory. This study was motivated by the belief that the experimental verification of theory should accompany the development of theory.

The system studied in this work consists of a jacket cooled continuous flow stirred tank reactor (CSTR), with a homogeneous liquid-phase, exothermic, irreversible chemical reaction. The reaction is the saponification of ethyl acetate. The optimal control problem considered here is the following:

Using the heat transfer coefficient between the reaction mixture and the coolant as the control variable, what is the control law which drives the reactor system from a given initial state to a specified final state in minimum

Martin A. Javinsky is with Chevron Research Company, Richmond, California. time?

This problem is significant in three practical applications: reactor start-up, changing from one steady state to another, and regulating specified final state conditions.

\section{PREVIOUS WORK}

The basic theoretical reference on the maximum principle is the book by Pontryagin and his co-workers (1). A good elementary account of the maximum principle can be found in the papers of Rozonoer (2).

While many significant applications of the maximum principle have been demonstrated in other engineering fields, it is only during the past few years that chemical engineers have begun to apply this mathematical theory to problems of chemical engineering importance. One of the first and most extensive of all studies in this area was accomplished by Aris and Siebenthal $(3,4)$. The work in this paper is based to a large extent on their study of the time-optimal control problem for a CSTR. Their studies were conducted with a simulated reactor system (with a general first-order reaction) on a digital computer; no experimental work was attempted.

Cotter and Takahashi (5) considered time-optimal control for a CSTR with feed flow rate and coolant flow rate 
as allowable control variables. The optimal control strategy was applied experimentally in one run of a continuous benzene nitration reactor. The optimal strategy maintained the feed flow rate at zero; therefore, the process was operated as a batch reactor during start-up. In the experimental run, it also turned out that the optimal strategy was a zero cooling water flow rate during the transient period. Thus, the overall optimal policy was to set the coolant and feed flow rates to zero and let the temperature in the reactor rise to the desired operating level. Then the flows were reset to maintain this level.

Latour and Koppel $(6,7)$ applied the maximum principle in a study of feedback, time-optimal switching controllers. An overdamped, second-order model with dead time was assumed to adequately represent the plant dynamics. The controllers were tested on plant simulations and on an experimental, two-tank, water heating, flow process. While claiming their techniques should be applicable to a broad class of process control problems, the authors admit (7) that processes such as a highly exothermic chemical reactor cannot be simulated satisfactorily by their generalized model.

Several other papers on the optimal control or optimization of chemical engineering systems (very often the system has been a CSTR) have appeared in the literature, but nearly all of them have been theoretical or computational in nature. A major objective of this study is to help fill the void caused by the lack of experimental studies in optimal control problems involving chemical engineering systems.

\section{FORMULATION AND SOLUTION OF THE PROBLEM}

To aid in the derivation of the state equations, a simple schematic sketch of the reactor is shown in Figure 1.

The reaction studied is the saponification of ethyl acetate:

$$
\mathrm{NaOH}+\mathrm{CH}_{3} \mathrm{COOC}_{2} \mathrm{H}_{5} \rightarrow \mathrm{CH}_{3} \mathrm{COONa}+\mathrm{C}_{2} \mathrm{H}_{5} \mathrm{OH}
$$

or, in general form

$$
A+B \rightarrow C+D
$$

The inlet concentrations and flow rates of reactants are equal. If the initial concentrations of all components in the reactor are consistent with the inlet concentrations and flow rates (that is, $c_{A}=c_{B}, c_{C}=c_{D}, c_{A}+c_{C}=c_{A_{0}}$ ), then one mass balance is sufficient to determine the concentration of any component. Of course, a heat balance is also required. The mass balance will be written for component A:

$$
\frac{d c_{A}}{d t}=\left(\frac{q_{A}}{V}\right) c_{A_{0}}-\left(\frac{q}{V}\right) c_{A}-A e^{-E / R T} c_{A}^{2}
$$

The reactor heat balance is given by

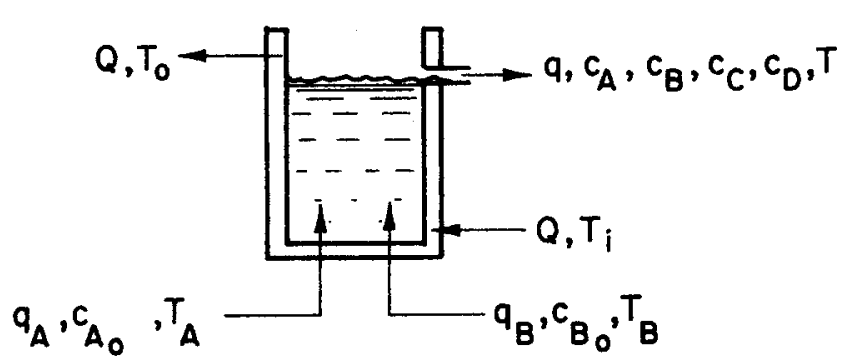

Fig. 1. Schematic sketch of reactor.
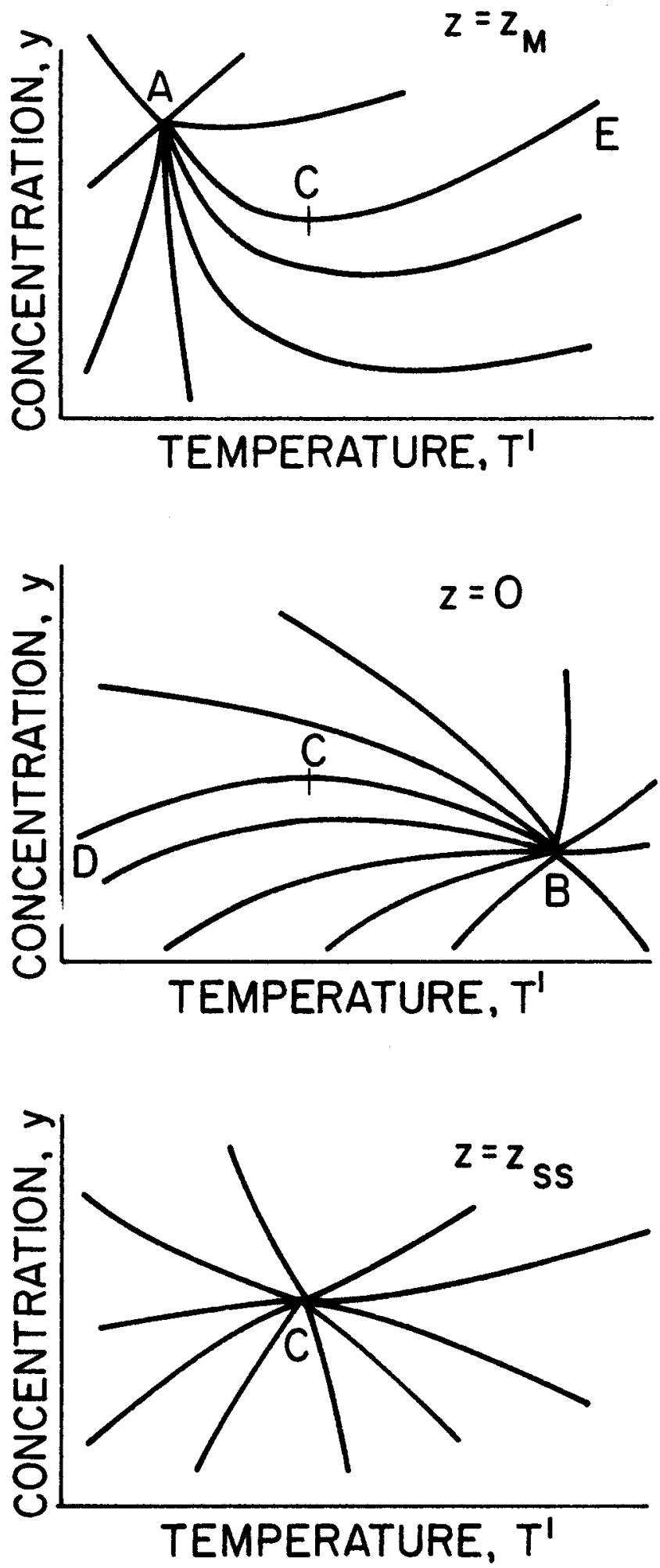

Fig. 2. Sketches of phase planes.

$$
\begin{aligned}
\frac{d T}{d t} & =\frac{q_{A}}{V} T_{A}+\frac{q_{B}}{V} T_{B} \\
& -\frac{q}{V} T-\frac{\Delta H}{\rho C_{p}} A e^{-E / R T} c_{A}^{2}-\frac{z}{V_{\rho} C_{p}}\left(T-T_{i}\right)
\end{aligned}
$$

The quantity $z$ is a modified overall heat transfer coefficient between the reactor and jacket fluids. The relationship between $z$ and the more commonly used overall heat transfer coefficient $U$ is given by

$$
z=U a /\left[1+U a / 2 \rho c_{p} Q\right]
$$


In addition to the assumptions of equal inlet flow rates and concentrations, the following assumptions were used in deriving the state equations:

1. The reactor is perfectly mixed.

2. Simple dynamics may be used to describe the heat transfer between the jacket and reactor fluids. The temperature driving force is based on the average jacket temperature, and this average temperature is in a quasi steady state condition with respect to the reactor fluid.

3. All fluids have the same densities and heat capacities, and these parameters are constants.

Furthermore, the feed flow rates, feed concentrations, feed temperatures, cooling water inlet temperature, heat of reaction, and reaction rate parameters will be considered as constants in the remainder of this analysis.

The state equations will be rendered dimensionless with the use of the following variables:

$$
\begin{gathered}
y=c_{A} /\left(\frac{q_{A}}{q} c_{A_{0}}\right) \\
t^{\prime}=\frac{q}{V} t \\
T^{\prime}=\frac{R}{E} T
\end{gathered}
$$

Then, Equations (3) and (4) become, respectively

$$
\begin{gathered}
\frac{d y}{d t^{\prime}}=1-y-\frac{V}{q} \frac{q_{A}}{q} c_{A_{0}} A e^{-1 / T^{\prime}} y^{2} \\
\frac{d T^{\prime}}{d t^{\prime}}=\frac{-\Delta H}{E} \frac{V}{q} \frac{R}{\rho c_{p}} \frac{\left(q_{A}\right)^{2}}{(q)^{2}} c^{2} A_{0} A e^{-1 / T^{\prime}} y^{2} \\
+\frac{q_{A}}{q} T_{A}^{\prime}+\frac{q_{B}}{q} T_{B}^{\prime}-T^{\prime}-\frac{z}{q \rho c_{p}}\left(T^{\prime}-T_{i}^{\prime}\right)
\end{gathered}
$$

The state variables are $y$ and $T^{\prime}$, and the control variable is $z$. In the physical system, the control variable is actually the cooling water flow rate $Q$. However, since there is a one-to-one correspondence between the variables $z$ and $Q$, $z$ may be used as the control variable in the mathematical model.

The control variable has upper and lower limits. The lower limit corresponds to adiabatic operation $(Q=0)$, and the upper limit corresponds to the maximum cooling water flow rate available. The control constraints are expressed mathematically in the following equation:

$$
0 \leq z \leq z_{M}
$$

The state variables are assumed to have initial conditions $y(0)$ and $T^{\prime}(0)$, where $y(0)$ is consistent with the inlet concentrations and flow rates.

It is desired to drive the system from the given initial condition to a steady state $\left(y_{s s}, T_{s s}^{\prime}\right)$ corresponding to a value of $z$, called $z_{s s}$, where $z_{s s}$ is chosen such that

$$
0<z_{s s}<z_{M}
$$

The physical system is designed to ensure that there is only one steady state $\left(y_{s s}, T_{s s}^{\prime}\right)$ corresponding to a given value of $z_{s s}$.

The optimization objective is to manipulate the control variable so that the transition from the initial condition to the steady state occurs in minimum time. In mathematical terms, the index of performance, $J$, is given by

$$
J=\int_{0}^{t_{f}} 1 d t=t_{f}
$$

To summarize, the problem is to find a control $z$ [subject to the constraints in Equation (11)] which drives the system [given by Equations (9) and (10)] from some initial condition $\left[y(0), T^{\prime}(0)\right]$ to the specified final state $\left(y_{s s}, T^{\prime}{ }_{s s}\right)$ so that $J$ (that is, $t_{f}$ ) is minimized.

Before we attempt to solve the problem, it is desirable to determine if a solution to the problem does indeed exist. Existence theorems for optimal control problems which include the type just formulated were presented by Markus and Lee (8). Siebenthal (3) applied these theorems to the general CSTR problem, and he showed that optimal controls do exist for the problem considered in this paper.

The solution to the optimal control problem formulated in this section can be obtained by application of the maximum principle. A statement of the maximum principle and its application in obtaining the solution to a generalized CSTR time-optimal control problem may be found in the paper by Siebenthal and Aris (4). The application of the maximum principle to the specific problem of this paper may be found in reference 9 .

The solution obtained by application of the maximum principle is a bang-bang control law. That is, the control variable $z$ assumes either its minimum value $(z=0)$ or its maximum value $\left(z=z_{M}\right)$ as the state of the system is transferred from an initial condition to the specified final condition. Furthermore, in this problem, the maximum principle permits no more than one switch of the control variable as the state of the system is transferred. Of course, once the specified final state is reached, the control variable must be switched once again (to $z_{s s}$ ) to maintain the steady state condition corresponding to $z_{s s}$. This final switch is not considered in the analysis with the maximum principle.

The optimal control policy determined with the maximum principle is an open loop control law. The initial control mode, the switching time during the transient period, and the time for the final switch to $z_{s s}$ are all specified for a particular initial condition.

In the problem considered here, it is possible to devise a closed loop optimal control law. Following are two facts which are used in determining the closed loop law:

1. The state equations are assumed to be autonomous. Theorems on the uniqueness of solutions for autonomous systems (10) guarantee that for a given value of the control variable $z$, trajectories in the concentration-temperature phase plane do not intersect at any point except the steady state point corresponding to $z$.

2. From the discussion above, it is known that during the transient period, the optimal control policy is bangbang. Furthermore, no more than one switch in the value of the control variable is permitted during the transient period.

Consider the concentration-temperature phase planes in Figure 2. Phase planes are sketched for values of $z$ equal to $z_{M}, o$, and $z_{s s}$, respectively. The steady state point (point $C$ ) on the $z_{s s}$ diagram is the desired final state. This point is also shown on the no cooling and full cooling diagrams. Since the system is autonomous, only one curve passes through point $C$ in each of the no cooling and full cooling diagrams. These curves are lettered $C D$ and $C E$. Curves $C D$ and $C E$ are redrawn on a concentration-temperature phase plane in Figure 3.

It will now be shown that curve $D C E$ is the optimal switching curve for the physical system. Consider a starting point such as point $F$. Suppose that the initial control mode is full cooling. If this control mode is used indefinitely, the system will eventually approach the steady state (point A) corresponding to full cooling. If the con- 


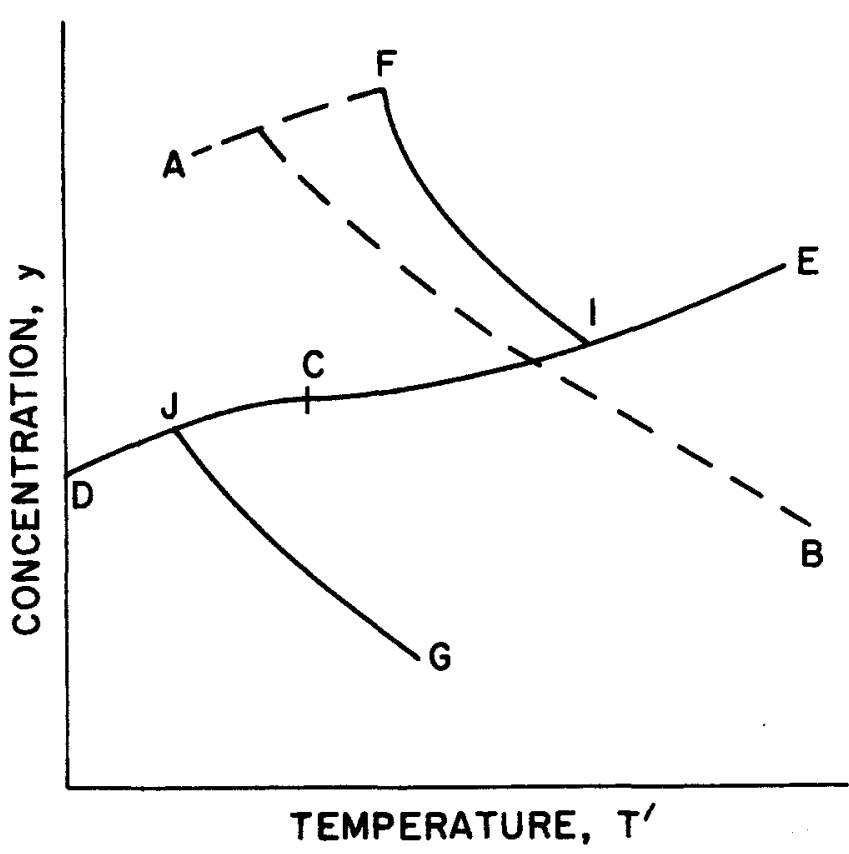

Fig. 3. Sketch of optimal switching curve and optimal trajectories.

trol variable is switched to zero before point $A$ is reached, the phase trajectory will move toward point $B$, the steady state corresponding to no cooling. As stated earlier, only one switch of the control variable is permitted. Hence, point $C$ cannot be reached if the initial control mode from point $F$ is full cooling.

Suppose now that the initial control mode from point $F$ is no cooling. The phase trajectory moves toward point $B$. Suppose that the control variable is switched to maximum cooling when the phase trajectory intersects curve $D E C$ (point I). Now, full cooling can be used until point $C$ is reached (at point $C$ the control variable must be set to $\left.z_{s s}\right)$. Thus, the system has been transferred from point $F$ to point $C$ with only one switch during the transient period. This policy satisfies the aforementioned requirements determined from the maximum principle.

An argument analogous to the one just presented can be used to show that the optimal control policy from a point such as point $G$ is to use full cooling until the trajectory intersects curve $D C E$ (point $J$ ) and to use no cooling until point $C$ is reached.

In the analogue computer studies described in a later section, it was found that the no cooling trajectories generated by integrating backwards in time from points on curve $C E$ covered the entire region above curve $D C E$. Similarly, the full cooling trajectories generated by integrating backwards in time from points on curve $C D$ covered the entire region below curve $D C E$.

The maximum principle, phase plane analysis, and existence theory have produced necessary and sufficient conditions which yield the following result. The unique optimal control policy from any point above curve $D C E$ is to use no cooling until curve $C E$ is reached and then to use full cooling until point $C$ is reached. The unique optimal control policy from any point below curve $D C E$ is to use full cooling until curve $D C$ is reached and then to use no cooling until point $C$ is reached.

Thus, the optimal control policy can be determined at any time from knowledge of the current state of the system. If a disturbance occurs, the optimal policy is applied according to the location of the new state resulting from the disturbance. This means that curve $D C E$ may be interpreted as a closed loop switching law.

Of course, some types of disturbances are not allowed in the preceding analysis. For the preceding analysis to be valid, at the end of the disturbance the state equations must be valid, and the new concentration must be consistent with the inlet feed flow rates and concentrations. For disturbances such as a step change in a feed temperature, a new switching curve must be determined, and a new steady state is achieved. In general, a new switching curve and a new steady state are obtained for every permanent change of the constant parameters in the state equations. However, for small changes of these parameters, the system performance may still be satisfactory with the use of the original switching curve. This is considered in detail in reference 9 .

\section{DESCRIPTION OF EQUIPMENT}

A schematic diagram of the jacketed reactor is shown in Figure 4. The relative dimensions of the reactor and its accessories (baffles and impeller) were determined from standard reactor designs. The effective volume is $3,200 \mathrm{ml}$.

A schematic diagram of the experimental flow system is shown in Figure 5. The cooling water flow rate is the control variable of interest in this paper. The main control valve consists of a combination of five solenoid valves and a globe valve. This system is used because of the bang-bang nature of the optimal control policy described in the previous section.

The globe valve is used to set the cooling water flow rate under normal flow conditions (the cooling water flowing through the jacket). To create a no cooling situation, five solenoid valves are energized to provide the following results:

1. The flow of water to the jacket is stopped.

2. The flow of water from the jacket manifold is stopped.

3. Two dump valves at the bottom of the jacket are opened.

4. A solenoid valve near the top of the jacket is opened for a few seconds (and then closed) to permit high pressure air to flush the water out of the jacket through the dump valves.

The entire dumping process takes approximately $5 \mathrm{sec}$. To reestablish a flow of cooling water through the jacket, the solenoid valves are de-energized.

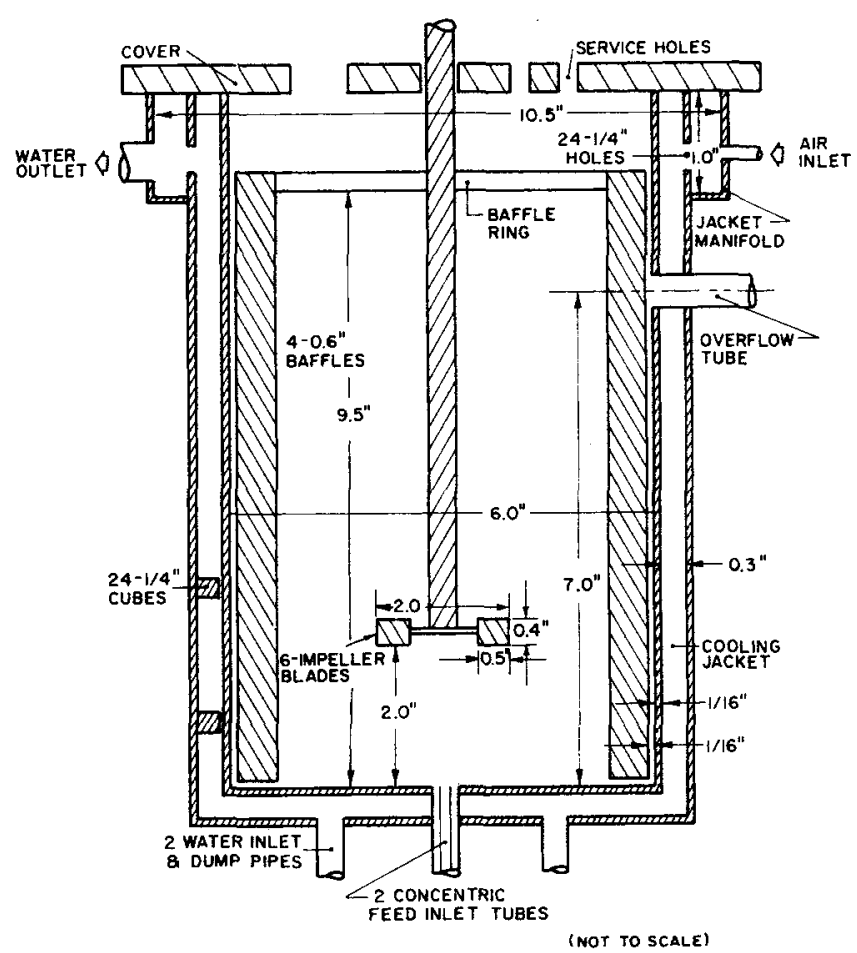

Fig. 4. Schematic diagram of reactor and accessories. 


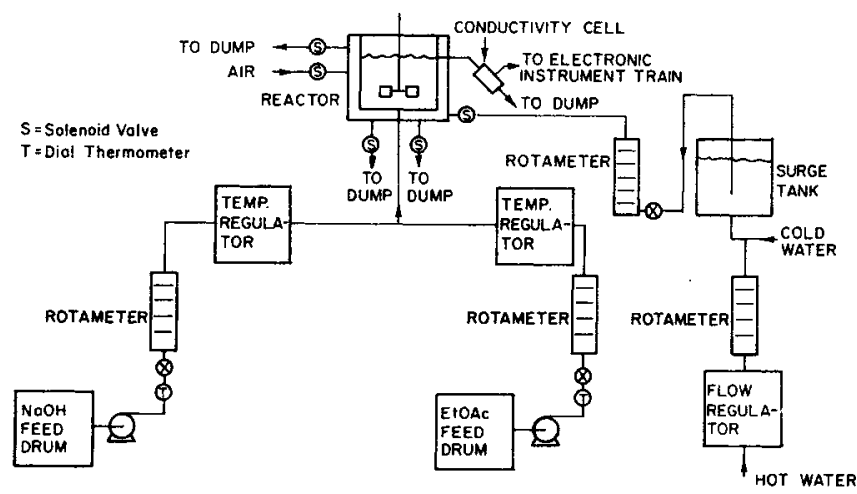

Fig. 5. Schemotic diagram of experimental flow system.

Temperatures of all streams and the reactor contents are measured with iron-constantan thermocouples. The concentration of the reactor effluent is determined with an on-line electrical conductivity cell. The reactor temperature and concentration signals are recorded continuously and simultaneously on an X-Y plotter to obtain the concentration-temperature phase plane.

Additional details on the design and construction of the equipment may be found in reference 9 .

\section{PRELIMINARY EXPERIMENTS}

Before the optimal control policy was applied to the experimental system, preliminary tests were conducted to verify assumptions made in deriving the model and to determine physical parameters of the system. Details of the procedures and results of these studies may be found in reference 9 . A brief summary is presented below:

1. Impulse-response tests showed that the reactor could be represented as a perfectly stirred tank.

2. Step response tests showed that heat transfer between the reactor and cooling jacket could be represented by first-order dynamics.

3. Reaction rate constants were calculated from steady state data by using the steady state form of Equation (3):

$$
k=A e^{-E / R T}=\frac{q_{A} c_{A_{0}}-q c_{A}}{V c_{A}^{2}}
$$

It was found that the extra conversion in the volume of piping between the reactor and the conductivity cell introduced an error in the calculation of the kinetic constants. To estimate the effect of the reactor exit pipeline, it was assumed that the line could be approximated by

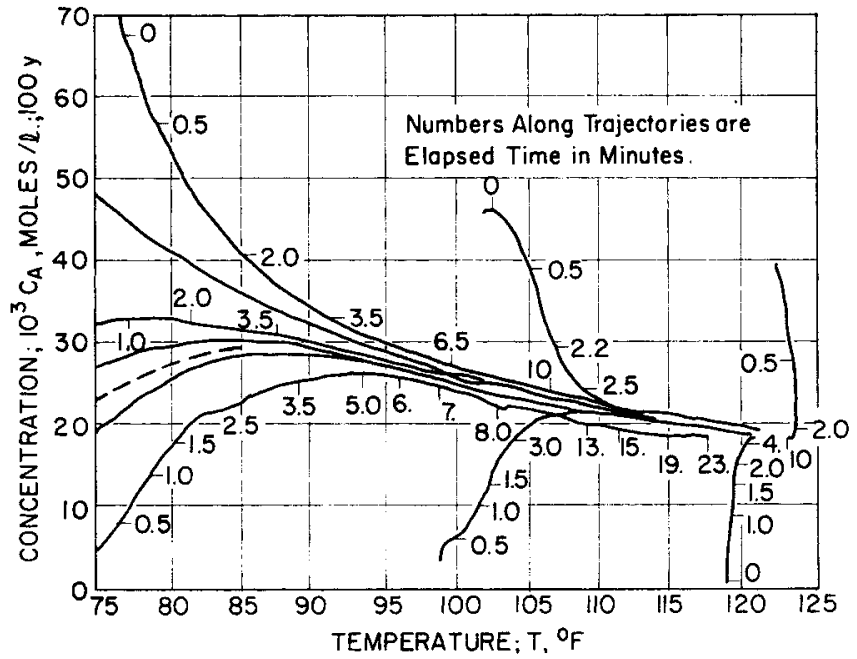

Fig. 7. No cooling phose plane. Experimental runs.

an isothermal plug flow reactor with a volume of $65 \mathrm{ml}$. If $c_{A}$ is the concentration of sodium hydroxide at the reactor outlet (the plug flow reactor inlet), then the concentration of sodium hydroxide at the conductivity cell (the plug flow reactor outlet) $c_{A, P F}$ is given by

$$
c_{A, P F}=\frac{c_{A}}{1+\tau_{P F} k c_{A}}
$$

Equation (15) can be solved for $c_{A}$ :

$$
c_{A}=\frac{c_{A, P F}}{1-\tau_{P F} k c_{A, P F}}
$$

Literature values for $k$ were calculated with values of $A$ and $E$ equal to $1.66 \times 10^{7} \mathrm{liter} / \mathrm{mole} / \mathrm{sec}$. and 11,210 cal./mole, respectively (11). The uncorrected and corrected results of the kinetic tests are shown in Table 1. The assumed plug flow reactor, while having a volume equal to only $2 \%$ of the stirred tank volume, significantly affects the calculation of the rate constants.

Since the literature values for the reaction rate constants are between the experimental values reported in columns 4 and 5 of Table 1 , and since the average absolute error between the experimentally determined values and the literature values is only $8 \%$, it was concluded that the experimental results obtained here confirm the values reported in the literature.

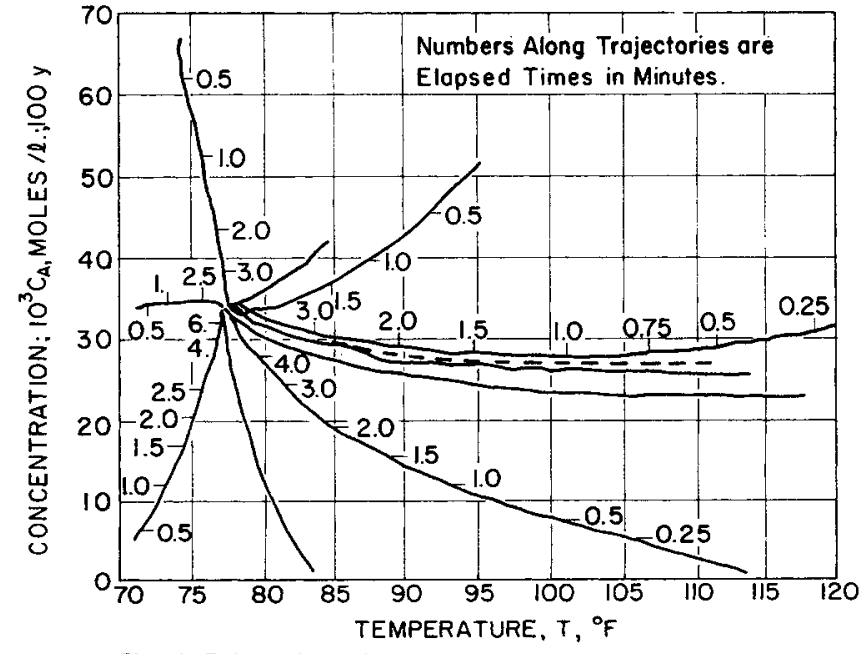

Fig. 6. Full cooling phase plane. Experimental runs.

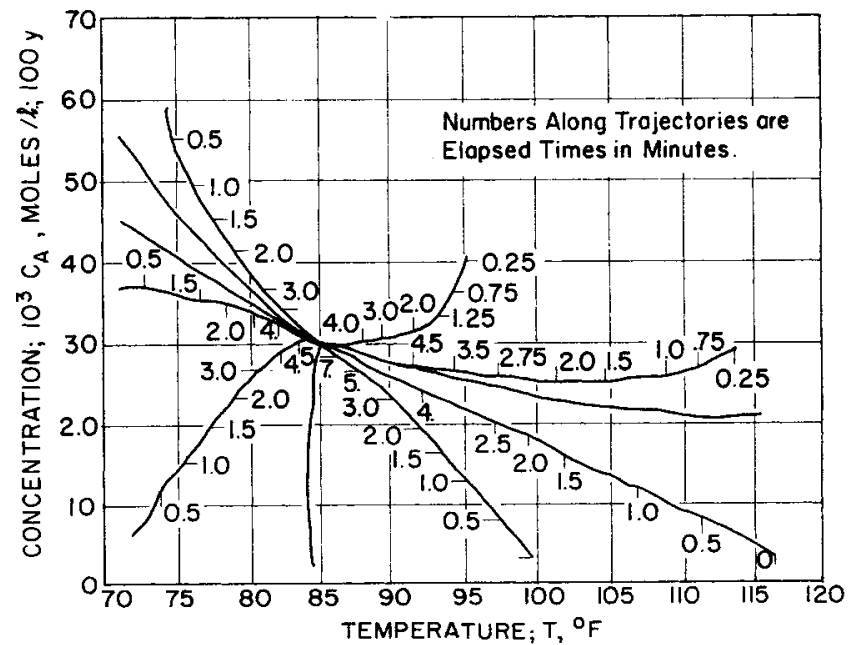

Fig. 8. Steady state cooling phase plane. Experimental runs. 


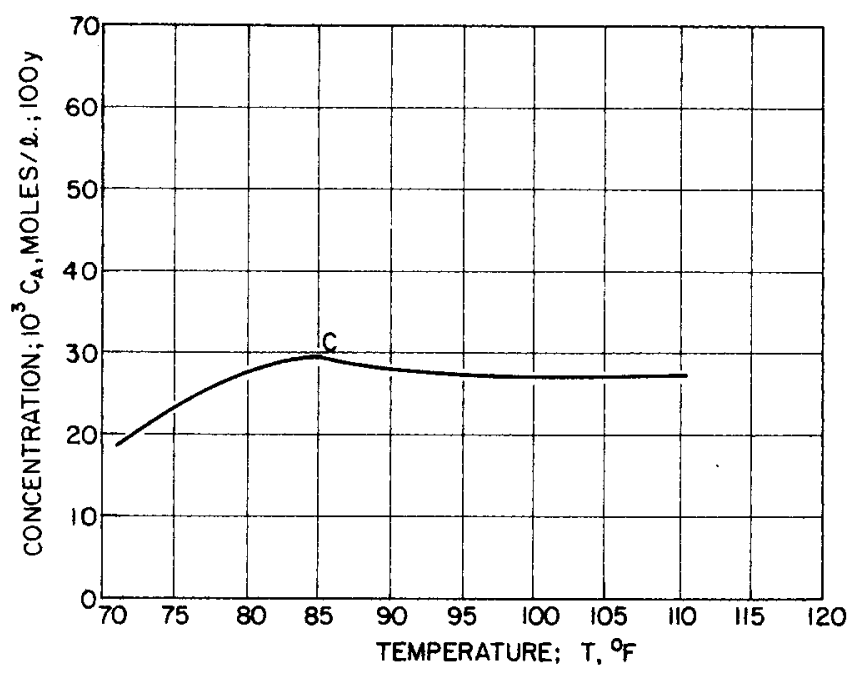

Fig. 9. Optimal switching curve from experimental runs.

\section{MAIN EXPERIMENTS}

Experimental phase plane trajectories were obtained for three cooling conditions: full, none, and steady state. From these phase trajectories, the closed loop optimal switching curve was determined by the method explained earlier, By using this optimal switching curve, minimum time experiments were conducted with various initial conditions. In some of these runs, when the state variables reached the desired terminal conditions, the cooling water flow rate was changed to the value required to maintain steady state conditions in the reactor. Several runs were conducted to determine the effects of early or late switching of the control variable.

\section{Procedure and Operating Conditions}

The concentration of sodium hydroxide and ethyl acetate in their respective feed streams was 0.2 moles/liter. In all runs, the flow rate of each feed stream was $200 \mathrm{ml}$./ min. The temperatures of both feed streams were regulated at $123^{\circ} \mathrm{F}$.

The cooling water temperature was regulated at $70.5^{\circ} \mathrm{F}$. The cooling water flow rates corresponding to full cooling and steady state cooling were 5 and 1 gal./min., respectively. The condition of no cooling was created by flushing all water out of the jacket with air by using the solenoid valve system described previously.

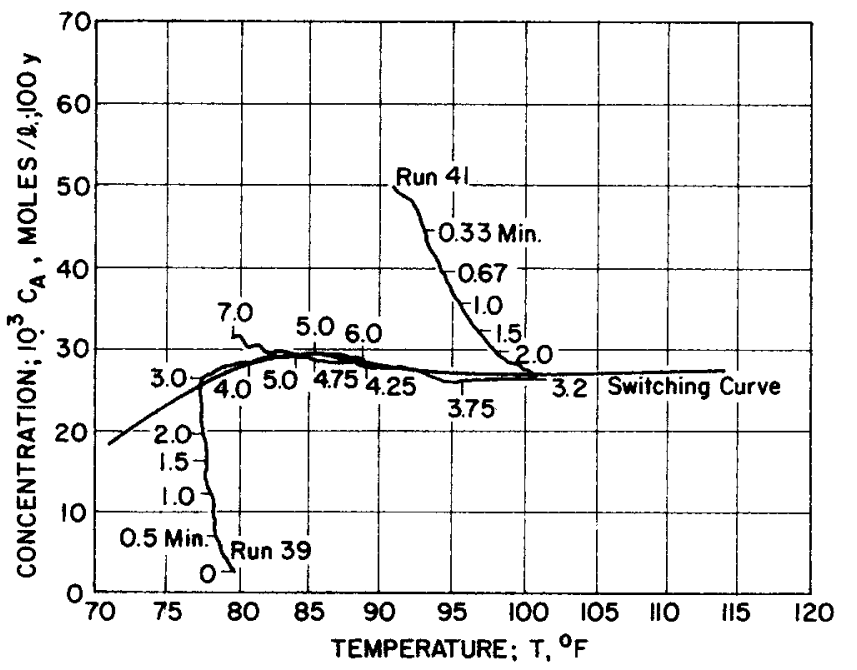

Fig. 10. Optimal trajectories. Experimental runs 39 and 41.

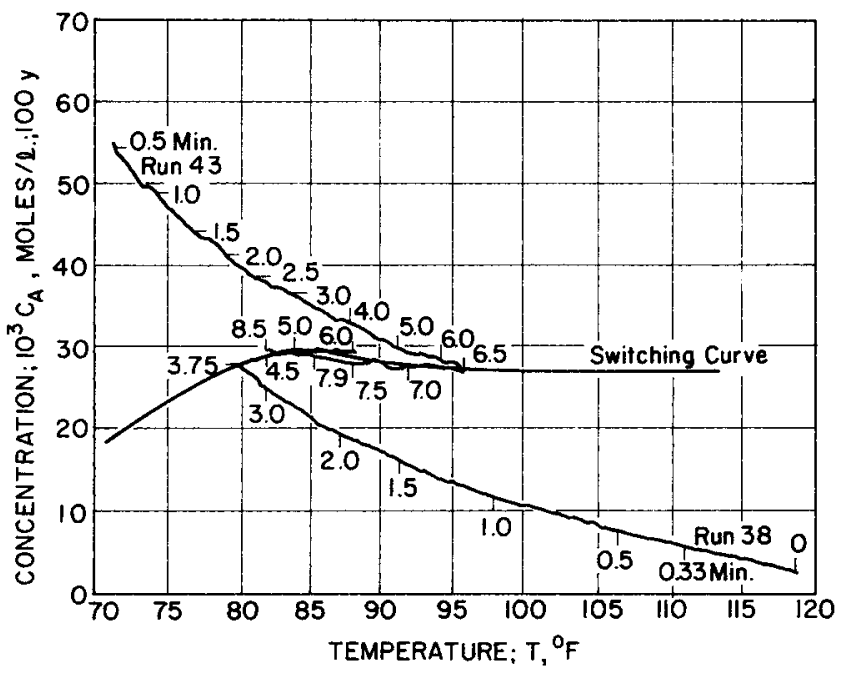

Fig. 11. Optimal trajectories. Experimental runs 38 and 43.

Before a run was started, the entire flow system was brought to a steady state condition. Then the reactor was emptied. The solutions to create the initial conditions in the reactor were poured into the reactor through a hole in the reactor cover. The concentrations of the components of the initial condition solution had to be consistent with the concentrations and flow rates of the feed streams. Thus, a typical initial solution consisted of $1,500 \mathrm{ml}$. of sodium hydroxide and $1,500 \mathrm{ml}$. of ethyl acetate, with each reactant having a concentration of 0.2 moles/liter before the solutions were combined (each reactant had a concentration of 0.1 moles/liter after the solutions were combined).

For the duration of the run, the concentration-temperature response was recorded on the X-Y plotter. In the optimal control runs, the optimal switching curve was drawn on the X-Y plotter graph paper before a run was started. During the run, when the phase trajectory reached the switching curve, the solenoid valve system was used to switch the cooling water flow rate.

\section{Discussion of Phase Plane Trajectories}

The experimental phase planes for full cooling, no cooling, and steady state cooling are shown in Figures 6, 7, and 8 , respectively. The steady state point (point $C$ ) in Figure 8 was defined as the desired terminal state (the final conditions of the state variables). The coordinates of point $C$ are $\left(82.5^{\circ} \mathrm{F}\right.$., 0.0295 moles/liter). The optimal switching curve was determined by locating the full cooling trajectory and the no cooling trajectory which pass through point $C$ in their respective phase planes. Since

\section{Table 1. Reaction Rate Constants}

\begin{tabular}{|c|c|c|c|c|c|}
\hline $\begin{array}{l}\text { Reactor } \\
\text { temp., } \\
{ }^{\circ} \mathrm{F} \text {. }\end{array}$ & $\begin{array}{c}c_{A, P F}, \\
\text { moles/ } \\
\text { liter }\end{array}$ & $\begin{array}{c}c_{A}, \\
\text { moles/ } \\
\text { liter }\end{array}$ & $\begin{array}{c}\text { Reaction } \\
\text { rate con- } \\
\text { stant with } \\
c_{A, P F} ; k, \\
\text { liter/ } \\
\text { (mole) } \\
\text { (min.) }\end{array}$ & $\begin{array}{c}\text { Reaction } \\
\text { rate con- } \\
\text { stant with } \\
c_{A} ; k, \\
\text { liter/ } \\
\text { (mole) } \\
\text { (min.) }\end{array}$ & $\begin{array}{c}\text { Literature } \\
\text { values for } k \text {, } \\
\text { liter/ } \\
\text { (mole) } \\
\text { (min.) }\end{array}$ \\
\hline 120.1 & 0.0193 & 0.0209 & 27.1 & 22.6 & 24.7 \\
\hline 80.9 & 0.0326 & 0.0339 & 7.92 & 7.19 & 6.94 \\
\hline 120.5 & 0.0192 & 0.0208 & 27.4 & 22.9 & 25.0 \\
\hline 110.1 & 0.0226 & 0.0242 & 18.9 & 16.1 & 18.2 \\
\hline 98.5 & 0.0258 & 0.0272 & 14.0 & 12.3 & 12.5 \\
\hline 109.1 & 0.0230 & 0.0246 & 18.2 & 15.5 & 17.6 \\
\hline 98.1 & 0.0260 & 0.0274 & 13.7 & 12.1 & 12.4 \\
\hline
\end{tabular}




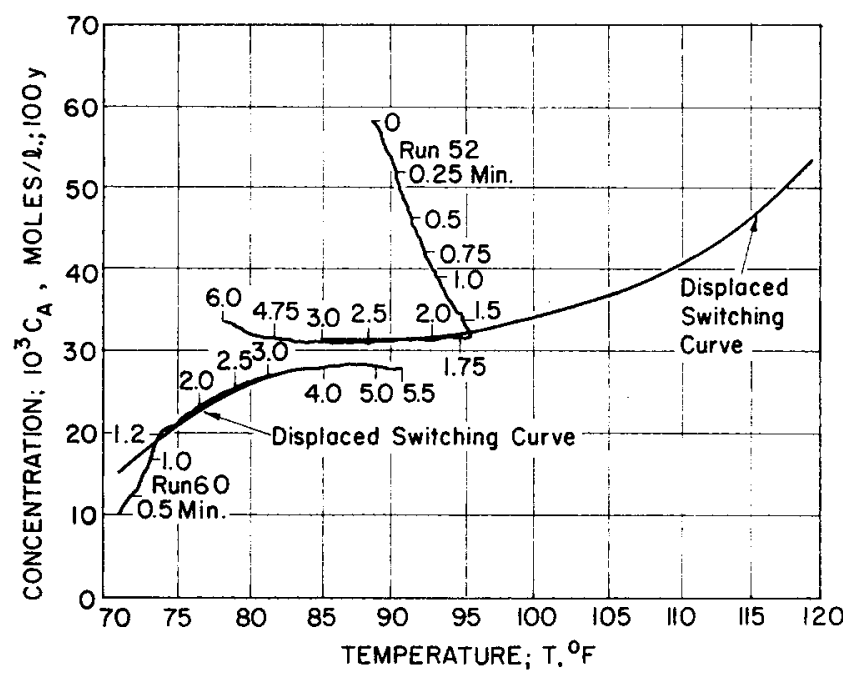

Fig. 12. Trajectories with early switching. Experimental runs 52 and 60.

none of the experimental trajectories in Figures 6 and 7 pass precisely through point $C$, the optimal switching curve was determined by sketching trajectories through point $C$. The trajectories were sketched so that they had the same shapes as their nearest neighboring experimental trajectories. The sketched trajectories are shown as dashed lines in Figures 6 and 7.

The resulting optimal switching curve is shown in Figure 9. The closed loop optimal control law states that all trajectories with initial conditions above the switching curve should have no cooling until the switching curve is reached and then full cooling until point $C$ is reached. All trajectories with initial conditions below the switching curve should have full cooling until the switching curve is reached and then no cooling until point $C$ is reached.

Several optimal trajectories redrawn from experimental data curves are shown in Figures 10 and 11. (The complete set of curves may be found in reference 9.) The elapsed times are marked along the trajectories. In all these runs, the trajectories were allowed to pass through and beyond the desired terminal state; that is, the cooling water flow rate was not changed to 1 gal./min. when the desired final state was reached. (In some other runs, when the phase trajectory reached the desired final state, the cooling water flow rate was changed from 0 or 5 to 1 gal./ min. to maintain the steady state. This final switch did not introduce any new problems, so these runs will not be discussed further.)

Note that the trajectories have some noise superimposed on the main response signal. The most probable sources of this noise are backmixing and fluid pulsations in the conductivity cell caused by slight pulsations of the liquid level in the reactor, air bubbles in the conductivity cell, and pen dragging effects in the conductivity recorder and $\mathrm{X}-\mathrm{Y}$ plotter, particularly as the trajectory approaches its steady state condition.

The noise associated with the trajectories introduced some uncertainty in the time to switch the control variable. In other words, there was some uncertainty in determining when a point on the phase trajectory precisely matched a point on the switching curve.

This switching uncertainty is one of the reasons that some of the optimal trajectories did not pass precisely through point $C$. For example, at the temperature corresponding to point $C$, the concentration of the trajectory of run 43 is 0.0005 moles/liter below the concentration corresponding to point $C$.

The sections of the optimal trajectories after the switch in the control variable should, of course, coincide with the sketched optimal switching curve. In general, at a given temperature, the concentration on an experimental trajectory was displaced from the concentration on the switching curve by an amount corresponding to an approximate average of $\pm 0.001 \mathrm{moles} / \mathrm{liter}$. These deviations can be explained in part by the switching uncertainties caused by the noise (as explained above) and by time delays in the switching procedure. However, it is believed that these deviations are more indicative of the reliability of the overall measuring and regulating equipment and the inherent difficulty of precisely reproducing transient data than they are of switching errors or any other welldefined errors.

To determine the advantage of using the optimal control scheme, the time to transfer from an initial condition to the final state with the optimal scheme was compared with the time to accomplish this transfer by using a cooling water flow rate of $1 \mathrm{gal} / \mathrm{min}$. for the entire run. Of course, with a cooling water flow rate of $1 \mathrm{gal} / \mathrm{min}$, the system reaches the final state only after an infinite time. Therefore, to make the time comparisons, a small circle around point $C$ was used as the final state.

Table 2 shows a comparison of optimal and uncon-

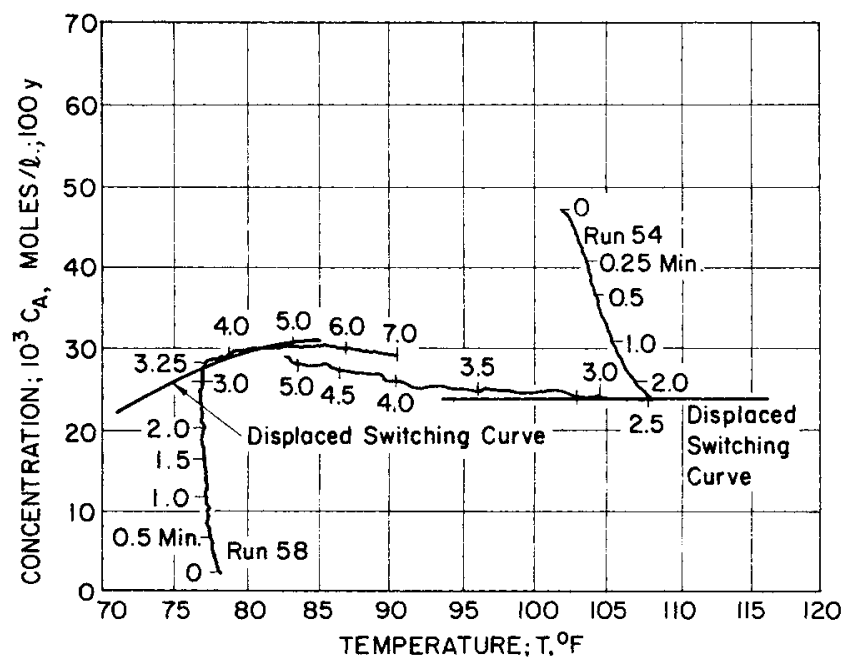

Fig. 13. Trajectories with late switching. Experimental runs 54 and 58.

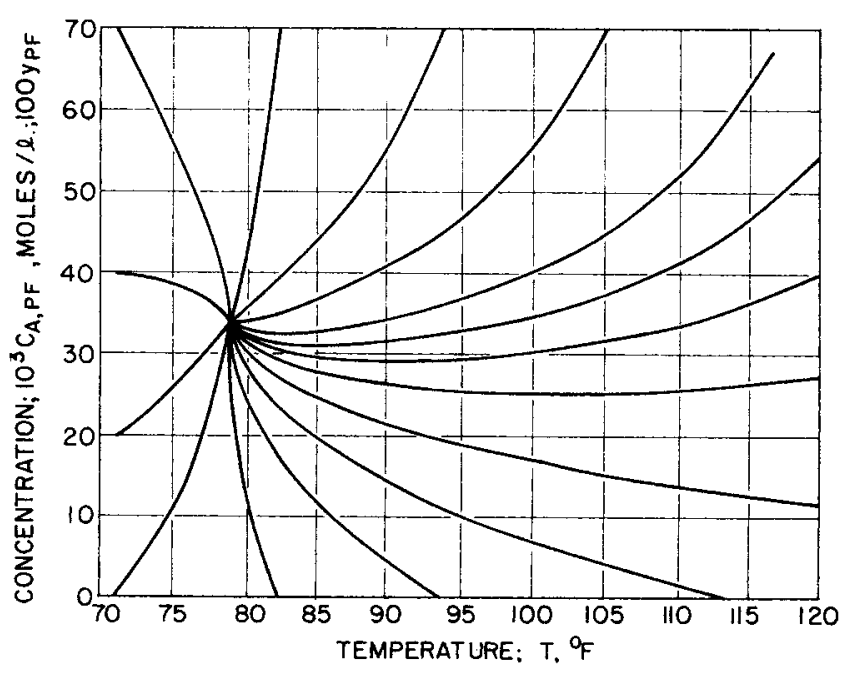

Fig. 14. Full cooling phose plane. Computer runs. 
Table 2. Time Comparisons for Uncontrolled and Optimally Controlled Runs
Initial conditions (temp. $T,{ }^{\circ} \mathrm{F}$, conc. $10^{3} c_{A}$, moles/liter)

\author{
Optimal run, time \\ to reach steady \\ state, $\min$.
}

Uncontrolled run, time to reach steady state, $\min$.

$\begin{array}{rrr}(75.0,62) & 7.5 & 10.0 \\ (72.8,5) & 4.5 & 9.5 \\ (116.4,4) & 5.6 & 10.0 \\ (112.5,30) & 2.7 & 9.5 \\ (93.8,45) & 4.5 & 9.5 \\ (84.1,14) & 3.7 & 6.5 \\ (71.1,55) & 7.5 & 11.0 \\ (107.9,26) & 1.3 & 8.5 \\ (72.5,5) & 4.5 & 9.5 \\ (85.2,5) & 4.7 & 6.5\end{array}$

trolled run times for various initial conditions. The time saved by the optimal control policy varies with the location of the initial conditions. The greatest savings of time occur for initial conditions at low concentrations and low temperatures and for initial conditions at high concentrations and high temperatures.

Figures 12 and 13 show trajectories obtained with the use of early or late switching times. As shown in the figures, the optimal switching curves were displaced to cause the switching errors.

Because the no cooling and full cooling curves for various initial conditions are close together in the vicinity of the desired terminal state (see Figures 6 and 7), the optimal switching policy guides the system close (concentration is $0.0295 \pm 0.002$ moles/liter when the temperature is $85.2^{\circ} \mathrm{F}$.) to the steady state point, even with the switching errors.

Comparison of run times (time to transfer from an initial state to the final state) with the optimal and displaced switching curves showed that the greatest differences in times occurred when the initial cooling mode was no cooling. In this case, a typical difference in run times was 90 sec. This time difference was approximately equal to the difference in switching times with the optimal and displaced switching curves (with the full cooling part of the switching curve used).

\section{ANALOGUE COMPUTER SIMULATION STUDIES}

The optimal control problem was simulated on an

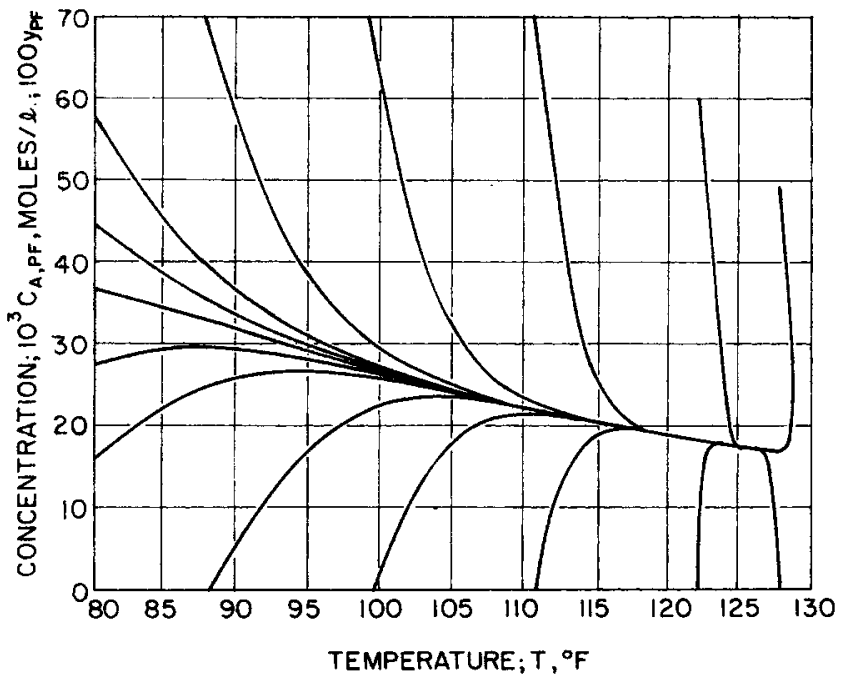

Fig. 15. No cooling phose plone. Computer runs. analogue computer to determine how well the experimental results could be predicted with the computer. Full cooling, no cooling, and steady state cooling phase planes obtained from the computer are shown in Figures 14, 15, and 16 , respectively. The phase trajectories obtained from the computer solutions have nearly the same shape as their experimental counterparts, which are shown in Figures 6 , 7 , and 8. The main discrepancies in the experimental and computer phase plane plots are the displacements of the steady state points. The coordinates of the experimental terminal state determined with the computer are $\left(87.3^{\circ} \mathrm{F}\right.$, 0.0300 moles/liter).

Optimal switching curves obtained from the computer solution and from the experimental data are shown in Figure 17. By comparing the experimental and computer phase plane plots and optimal switching curves, it can be seen that the main discrepancies between the experimental and computer runs are the displacement of the steady state points and the displacement of the full cooling part of the optimal switching curve. The first error contributes significantly to the second error. In other words, the full cooling computer trajectory (corrected for the assumed plug flow error) which passes through the point $\left(85.2^{\circ} \mathrm{F}\right.$, $0.0295 \mathrm{moles} / \mathrm{liter}$ ) is closer to the full cooling part of the experimental switching curve than is the trajectory which passes through the point $\left(87.3^{\circ} \mathrm{F}\right.$, 0.0300 moles/ liter).

The errors in the steady state points are caused by uncertainties in the model and the values of the model parameters as well as uncertainties in the experimental measuring system. In addition to the effect of the steady state displacement, minor wall effects on the heat transfer dynamics might have caused the error in the full cooling part of the optimal switching curve. All experimental full cooling trajectories were generated by switching from no cooling to full cooling after the initial condition solution had been poured into the reactor. Thus, the wall effect on the dynamics would cause the temperature of the reactor solution to stay too warm for too long a period of time. This, in turn, would cause the conversion of reactants to be greater than the predicted conversion.

From information discussed in the last section on the experimental runs with late switching of the control variable, it can be seen that the discrepancy in the full cooling part of the experimental and computer switching curves does not have great significance in this study. Because the full cooling trajectories converge rapidly in the vicinity of the specified final state, the overall performance of the

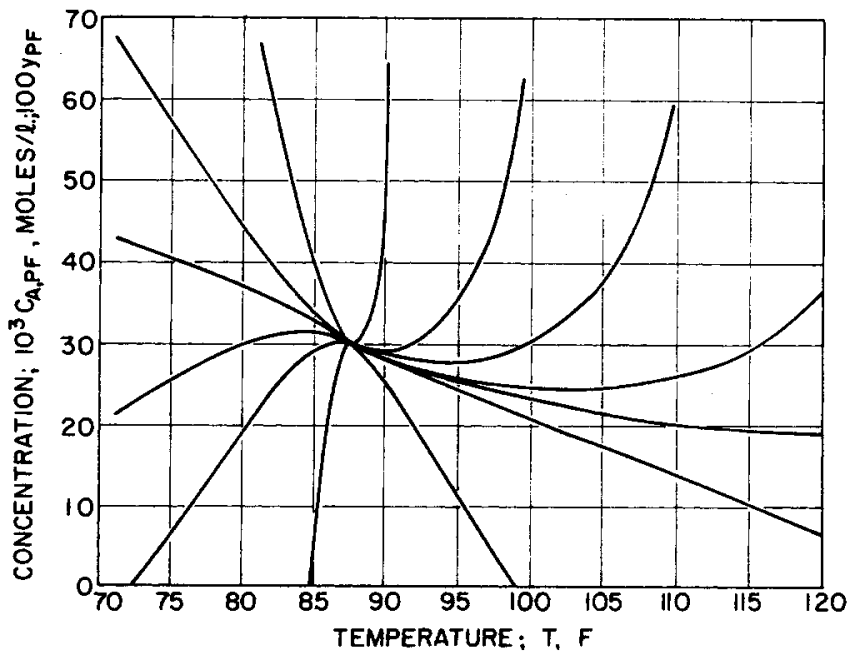

Fig. 16. Steady state cooling phase plane. Computer runs. 


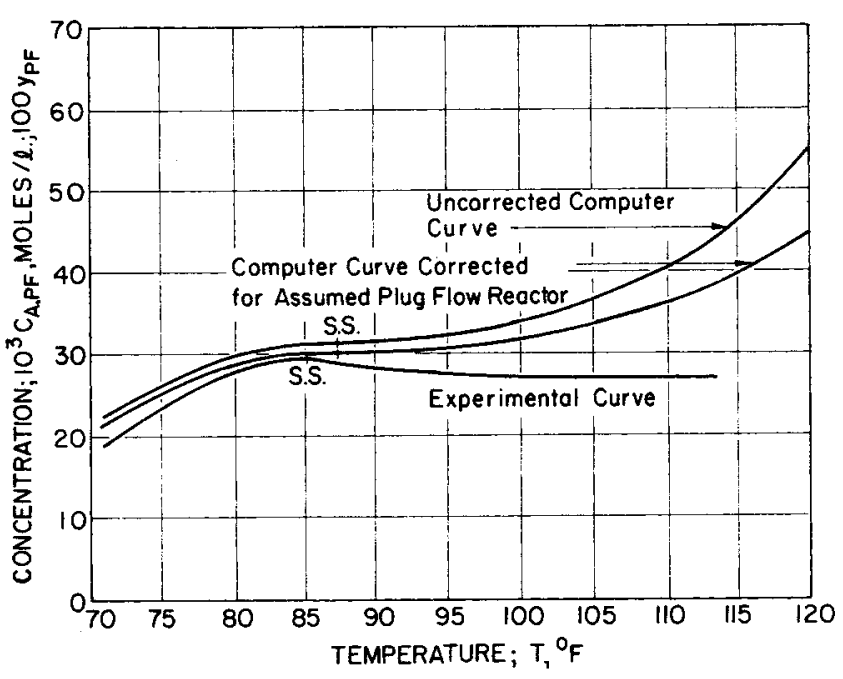

Fig. 17. Optimal switching curves. Computer and experimental runs.

experimental system would probably be acceptable if the computer switching curve (corrected for the extra conversion in the assumed plug flow reactor) were used instead of the experimental switching curve.

\section{CONCLUSIONS}

The overall performance of the experimental system agreed very well with the performance of the simulated system. The main differences in the experimental and simulated systems were the displacement of the final state and the displacement of the optimal switching curve. These differences were attributed to uncertainties in the model and the values of the model parameters as well as uncertainties in the experimental measuring system. The extra conversion of reactants in the pipeline between the reactor and the electrical conductivity cell produced a nonrandom and unforeseen measurement error. This is just one example of why experimental data should be used to check the results of simulation studies.

The time saved by using the optimal control policy (rather than no control at all) to transfer the experimental system from an initial condition to the final state varied from 25 to $85 \%$ of the transient time obtained with the uncontrolled response. The amount of time saved varied with the location of the initial condition in the phase plane. The greatest savings of time occurred for initial conditions at high conversions and low temperatures and for initial conditions at low conversions and high temperatures.

The results obtained in this study show that the maximum principle can be successfully applied to solve the time-optimal problem for a laboratory sized reator. Further studies should be conducted in pilot sized and plant sized reactors to determine whether or not the performance of a large system subjected to the optimal control policy is significantly different from the performance of a small system. Imperfect mixing in the reactor and high order heat transfer dynamics would be the most probable causes of any differences that would be observed in the performance of the large system.

\section{NOTATION}

$a=$ area for heat transfer, sq.ft.

$A=$ frequency factor in Arrhenius rate expression, liter $/ \mathrm{mole} / \mathrm{sec}$. $\left(1.66 \times 10^{7}\right)$

$c=$ concentration, moles/liter
$c_{A_{0}}=$ concentration of sodium hydroxide in feed stream, moles/liter (0.2)

$c_{A, P F}=$ concentration of sodium hydroxide corrected for extra conversion in assumed plug flow reactor, moles/liter

$c_{\mathrm{B}_{0}}=$ concentration of ethyl acetate in feed stream, moles/liter (0.2)

$c_{P}=$ specific heat, cal./g. $/{ }^{\circ} \mathrm{C}$. (1.0)

$e \quad=$ base of natural logarithms

$E=$ energy of activation, cal./mole $(11,210)$

$\Delta H=$ heat of reaction, cal./mole $(-17,700)$

$J=$ index of performance

$k=$ reaction rate constant, liter/mole/sec.

$q=$ feed How rate, $\mathrm{ml} . / \mathrm{min}$. (200)

$Q \quad=$ cooling water flow rate, gal./min.

$\boldsymbol{R}=$ gas constant, cal. $/ \mathrm{mole} /{ }^{\circ} \mathrm{C}$. (1.99)

$t=$ time, sec.

$t_{f}=$ final time, sec.

$T=$ temperature, ${ }^{\circ} \mathrm{F}$.

$T_{i}=$ cooling water inlet temperature, ${ }^{\circ} \mathrm{F}$. (70.5)

$T_{o}=$ cooling water outlet temperature, ${ }^{\circ} \mathrm{F}$.

$U=$ overall heat transfer coefficient, B.t.u./hr./sq.ft./ ${ }^{\circ} \mathrm{F}$.

$V \quad=$ volume of reactor, $\mathrm{ml} .(3,200)$

$y=$ fraction of unconverted sodium hydroxide

$z=$ control variable (modified heat transfer coefficient), B.t.u. $/$ hr. $/{ }^{\circ} \mathrm{F}$.

$\rho \quad=$ density, g./ml. (1.0)

$\tau_{P F}=$ space time of plug flow reactor, min.

\section{Subseripts}

$A=$ sodium hydroxide

$B \quad=$ ethyl acetate

$C$ = sodium acetate

$D \quad=$ ethyl alcohol

$f \quad=$ final value

$M \quad=$ maximum value

$P F \quad=$ plug flow, value corrected for assumed plug flow reactor

ss $\quad=$ steady state value

\section{Superseripts}

$=$ rescaled variable

$=$ time derivative

\section{LITERATURE CITED}

1. Pontryagin, L. S., V. G. Boltyanskii, R. V. Gamkrelidze, and E. F. Mischenko, "Mathematical Theory of Optimal Processes," Wiley, New York (1962).

2. Rozonoer, L. I., J. Automation Remote Control, 20, No. 10, 1288 (1959); Ibid., No. 11, 1405; Ibid., No. 12, 1517.

3. Siebenthal, C. D., Ph.D. thesis, Univ. Minn., Minneapolis, (1963).

4. 729 ( 1964 ); Ibid., 747.

5. Cotter, J. E., and Y. Takahashi, Chem. Eng. Progr. Symposium Ser. No. 46, 59, 119 (1963).

6. Latour, P. R., Ph.D. thesis, Purdue Univ., West Lafayette, Ind. (1966).

7. — , and L. B. Koppel, Ind. Eng. Chem. Fundamentals, 4, No. 4, 463 ( 1965 ).

8. Markus, L., and E. B. Lee, Arch. Rat. Mech. Analysis, 8, No. 1, 36 (1961).

9. Javinsky, M. A., Ph.D. thesis, Univ. Mich., Ann Arbor (1967).

10. Coddington, E. A., and N. Levinson, "Theory of Ordinary Differential Equations," McGraw-Hill, New York (1955).

11. Moelwyn-Huges, E. A., "Kinetics of Reaction in Solution," 2 ed., Clarendon Press, Oxford, England (1947).

Manuscript received May 8, 1968; revision received February 3, 1969; paper accepted February 5, 1969. Paper presented at AIChE St. Louis meeting. 